\title{
Evaluating the role of small particle hyaluronic acid fillers using micro-droplet technique in the face, neck and hands: a retrospective chart review
}

This article was published in the following Dove Press journal:

Clinical, Cosmetic and Investigational Dermatology

\author{
Andreas Nikolis ${ }^{1-4}$ \\ Kaitlyn M Enright ${ }^{1,3-5}$ \\ 'Victoria Park Medispa, Montreal, \\ QC, Canada; ${ }^{2}$ Department of Plastic \\ Surgery, Université de Montreal, \\ Montreal, QC, Canada; ${ }^{3}$ VP Research \\ Unit, Montreal, QC, Canada; ${ }^{4}$ Erevna \\ Innovations Inc., Montreal, QC, \\ Canada; ${ }^{5}$ Department of Experimental \\ Surgery, McGill University, Montreal, \\ QC, Canada
}

Background: Loss of the viscoelastic properties of the skin is a primary sign of aging and contributes to the appearance of wrinkles. Hyaluronic acid (HA) fillers are one of the most commonly used treatments for age-related soft-tissue reduction and volume loss. Evidence is also emerging that HA fillers rejuvenate the skin.

Methods: A retrospective chart review was completed on 20 subjects treated with small particle HA (SP-HA), to investigate its effects on skin properties. Subjects having received three treatments in the face, neck, and/or hands were considered in the analyses. Skin hydration, trans-epidermal water loss (TEWL), and $\mathrm{pH}$ were assessed at baseline (injection \#1), Week 4 (injection \#2), Week 8 (injection \#3), and Week 12 (follow-up).

Results: Treatment with SP-HA significantly improved hydration levels in the face, neck, and hands. Significant results were seen in the face following the first three treatments, with subjects moving up to the next hydration level (ie, hydration went from dry to moisturized) and by the second treatment in the neck and hands. TEWL scores on the face and neck remained within healthy values throughout all visits. At baseline, TEWL scores on the hands were within critical condition and after three injections they recuperated to healthy values, while $\mathrm{pH}$ values remained within the normal range throughout treatment.

Conclusion: A treatment regimen consisting of three SP-HA injections was safe and well tolerated. SP-HA use demonstrated a hydrating effect while positively impacting the skin's ability to retain moisture.

Keywords: restylane, skinboosters, hyaluronic-acid, HA, skin, hydration

\section{Background}

The use of injectable hyaluronic acid (HA) fillers for facial esthetics is among the most frequent non-surgical cosmetic procedure performed in the US, ${ }^{1,2}$ with more than a million HA procedures performed annually. ${ }^{3}$ To provide more tailored treatment options to physicians and subjects, a small-particle HA (SP-HA) was developed to address skin revitalization and was originally launched in Europe in 2004 (Restylane ${ }^{\circledR}$ Vital; Galderma, Uppsala, Sweden). Its low degree of cross-linking gives SP-HA viscoelastic properties that make these products especially pliable and moldable, which is important for achieving an optimal esthetic outcome with injections into the dermal layer of the skin. ${ }^{4}$

A SP-HA has recently been approved in Canada as a skin booster for rejuvenation of the face, neck, and hands (Restylane ${ }^{\circledR}$ Skinboosters Vital $20 \mathrm{mg} / \mathrm{mL}$ and Vital Light $12 \mathrm{mg} / \mathrm{mL}$; Galderma). Injections of SP-HA have been shown to be
Correspondence: Andreas Nikolis Erevna Innovations Inc., 973 Dunsmuir Montreal, QC, H3R3AI, Canada Email anikolis@vicpark.com 
efficacious and well tolerated for treating skin quality., $1,5-9$ SP-HA is typically injected using an intradermal microdroplet technique that deposits small amounts of product $(\mathrm{eg}, 10 \mu \mathrm{L})$ throughout the treatment area. However, SP-HA can be injected using multiple different techniques (eg, threading, fanning, puncture, etc), depending on the injector's preference after clinical assessment. While the ability of HA to improve the appearance of fine lines is well established, clinical observation also suggests that SP-HA may rejuvenate the skin by increasing hydration to the treatment area. Skin requires sufficient moisture for biomechanical functions which can impact normal skin physiology. As such, adequate hydration is paramount to establishing skin health. Furthermore, dehydration can cause dry, flaky, and tight skin that increases the appearance of age. Increasing moisture and hydration can therefore rejuvenate the skin by improving the appearance and health of skin. The mechanism of action is presumably HA's known hydrophilic properties. However, studies published to date have reported varied results on the efficacy of SP-HA in improving skin hydration. ${ }^{5,69}$ The difficulty in accurately measuring hydration may account for these variations in findings. For example, environmental influences (eg, room temperature, humidity) and patient behavior (eg, smoking, alcohol consumption) could account for the mixed findings found in the relevant literature. The authors of this article thus set out to investigate the following research question under standardized conditions: Do micro-aliquot injections of SP-HA increase cutaneous hydration in the face, neck, and hands in a reproducible and safe manner?

This article presents the first reported safety and efficacy evaluation of SP-HA in a Canadian population, as well as the impact of SP-HA on various skin parameters (ie, hydration, $\mathrm{pH}$, and trans-epidermal water loss [TEWL]) following treatment of the face, neck, and hands.

\section{Study design}

The following study received unconditional approval from the centralized Institutional Review Board "Veritas IRB". The protocol's tracking number is: 16139-12:58:3811-04-2017. A waiver of informed consent was granted by the IRB, in accordance with normative guidelines and Health Canada regulations. The procedures in this study conform to the guiding principles of the Declaration of Helsinki.

All active patient charts within one center were reviewed for inclusion criteria in a retrospective chart review to identify subjects treated with SP-HA in a skin health improvement program. As part of the program, it was standard for the center to collect data on skin parameters such as hydration, $\mathrm{pH}$, and TEWL at each visit. Patient selection was based on a convenience sampling method. Identifiable medical charts were coded and a blinded study coordinator used the nonidentifiable coded charts to evaluate eligibility. All research procedures were performed on non-identifiable patient data. Females between the ages of 30 and 75 years who underwent SP-HA treatments to improve skin smoothness, appearance, and elasticity in the lower cheek/jawline, neck, and/ or dorsal hands were eligible. Exclusion criteria included known hypersensitivity to HA fillers, lidocaine, amide local anesthetics, or streptococcal proteins; a history of bleeding disorders, porphyria, or keloids; and treatment with therapies thought to be confounding (ie, other HA fillers in the same areas in the last 6 months). Subjects having received three treatments, between 4-5 weeks apart, were considered in the final analyses. A total of four visits were assessed for each patient: Visit 1 - Baseline (injection \#1), Visit 2 - Week 4 (injection \#2), Visit 3 - Week 8 (injection \#3), and Visit 4 Week 12 (follow-up).

Charts meeting the following criteria were excluded: males, as gender-related differences in the skin's matrix has been shown to have an effect on the viscoelastic properties considered in this review; ${ }^{10}$ subjects under 30 or over 75 years old, due to age-related differences in skin properties; ${ }^{11} \mathrm{a}$ Fitzpatrick skin type $\mathrm{V} ;{ }^{12}$ smokers, as smoking can dehydrate the skin; ${ }^{13}$ refusal to have signed the photo release consent form; and concomitant medications or diseases that affect skin hydration or cause an increased level of swelling or bruising. ${ }^{14}$ Only subjects with complete Corneometer, $\mathrm{pH}$, and TEWL data sets were reviewed. Figure 1 displays a flow chart outlining the protocol methods.

Condition of measurements: The room which housed the devices measuring the various skin parameters remained constant as numerous evaluations are carried out requiring multiple daily recordings of temperature (fixed between $22^{\circ} \mathrm{C}$ and $24^{\circ} \mathrm{C}$ ) and humidity (between $20 \%$ and $30 \%$ ). As with all subjects who undergo skin measurements at the center, subjects were adapted to room conditions 20-30 minutes prior to testing. This was usually the time required to fill in the patient diaries and undergo photography sessions. Measurements were performed within the same 4-hour window to exclude diurnal variations. Skin measurements were taken at each visit, just prior to receiving their next scheduled injections.

Injection technique: Areas of injection were preconditioned with ice for 5 minutes prior to treatment and for 10-15 minutes following injections. The areas injected were standardized and included bilateral measurements for: 1) 


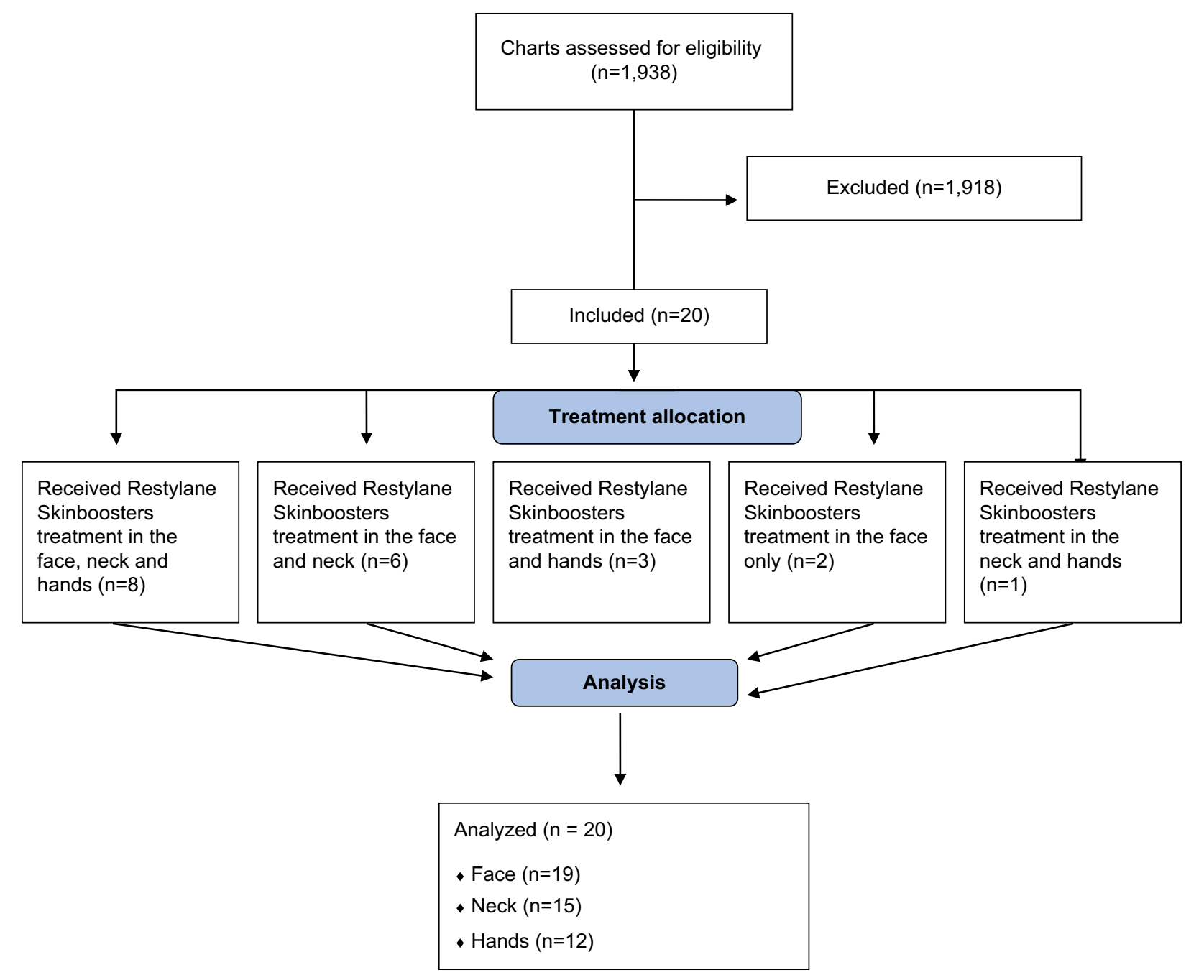

Figure I Study flow chart describing protocol methods.

the face (cheek region) with measurements $3 \mathrm{~cm}$ lateral to the modiolus; 2) the neck with measurements $4 \mathrm{~cm}$ lateral to the midline, within each prominent horizontal wrinkle at a point equidistant from the midline; and 3) the hands with injections $1 \mathrm{~cm}$ proximal to the metacarpal phalangeal joints and $1 \mathrm{~cm}$ distal to the extensor retinaculum. Face, neck, and hands were injected at $1 \mathrm{~cm}$ intervals. Hand injections required the use of a skin tenting technique. Injections were performed on either side of the tendons, with special care not to inject the tendons, tendon sheath, and vascular structures. The maximal volumes included 0.5 $\mathrm{mL}$ per each cheek, $0.3 \mathrm{~mL}$ for each side of the neck, and $0.5 \mathrm{~mL}$ for each hand.

Outcome measures: The primary outcome of interest was the measure of skin hydration as evaluated by the Corneometer ${ }^{\circledR} \mathrm{CM} 825$ using the Multi-Probe Adapter (MPA) System by Courage + Khazaka (Cologne, Germany). The
Corneometer measures the skin moisture content in arbitrary units between 0 and 130, with $<30$ indicating very dry; 30-40 dry; and $>40$ sufficiently moisturized. Individual values assessed per area were represented by an average of three Corneometer determinations. Other measures evaluated on an exploratory basis included $\mathrm{pH}$ and TEWL. TEWL was evaluated using the Tewameter ${ }^{\mathbb{B}}$ TM 300 , Courage + Khazaka (Cologne, Germany). The Tewameter measures the rate of water lost through the skin in $\mathrm{g} / \mathrm{h} / \mathrm{m}^{2}$ and is an estimate of the skin's ability to retain moisture. Higher TEWL values indicate greater water loss and are consistent with increased damage of the barrier function of the stratum corneum. A TEWL score below 25 is indicative of healthy skin, as per manufacturer guidelines. ${ }^{17}$ Individual TEWL values were represented by an average of 20 determinations. TEWL scores were considered for safety analyses, as treatment with SP-HA was not expected to cause an increase in water 
loss through the stratum corneum. $\mathrm{pH}$ was assessed using the pHmeter ${ }^{\circledR}$ PH 905, Courage + Khazaka (Cologne, Germany) and was considered for safety analyses, as treatment with SP-HA was not expected to cause a pH imbalance (eg, a normal skin $\mathrm{pH}$ range is between 4.0 and 7.0). ${ }^{15-17}$ Data on adverse events (AEs) were also collected, as well as 2D and $3 \mathrm{D}$ photos.

\section{Statistical analyses}

Descriptive statistics such as the mean, SDs, and mean differences from baseline were collected on all skin variables assessed (ie, hydration, $\mathrm{pH}, \mathrm{TEWL}$ ), at each visit. A two-tailed paired-sample $t$-test was used to compare means between all visits. Significance for all analyses was set at $<0.05$.
The group means for hydration were categorized based on the Corneometer results (ie, $<30$ very dry; 30-40 dry; and $>40$ sufficiently moisturized), in accordance with the hydration values defined and validated by the manufacturer ${ }^{17}$ at each visit. Group mean TEWL scores and $\mathrm{pH}$ was categorized as falling within healthy/normal or critical ranges. ${ }^{17}$

\section{Results}

Of all patient charts reviewed $(\mathrm{N}=1,938), 20$ met all eligibility requirements and were considered for evaluation. The included charts were comprised of women with a mean age of 58.92 years (range: 46-70, SD: 9.27). Nineteen subjects received SP-HA treatments in the face (Figures 2-4), 15 received treatments in the neck, and 12 received treatments in the hands (Figures 5 and 6). The majority of the 20 subjects
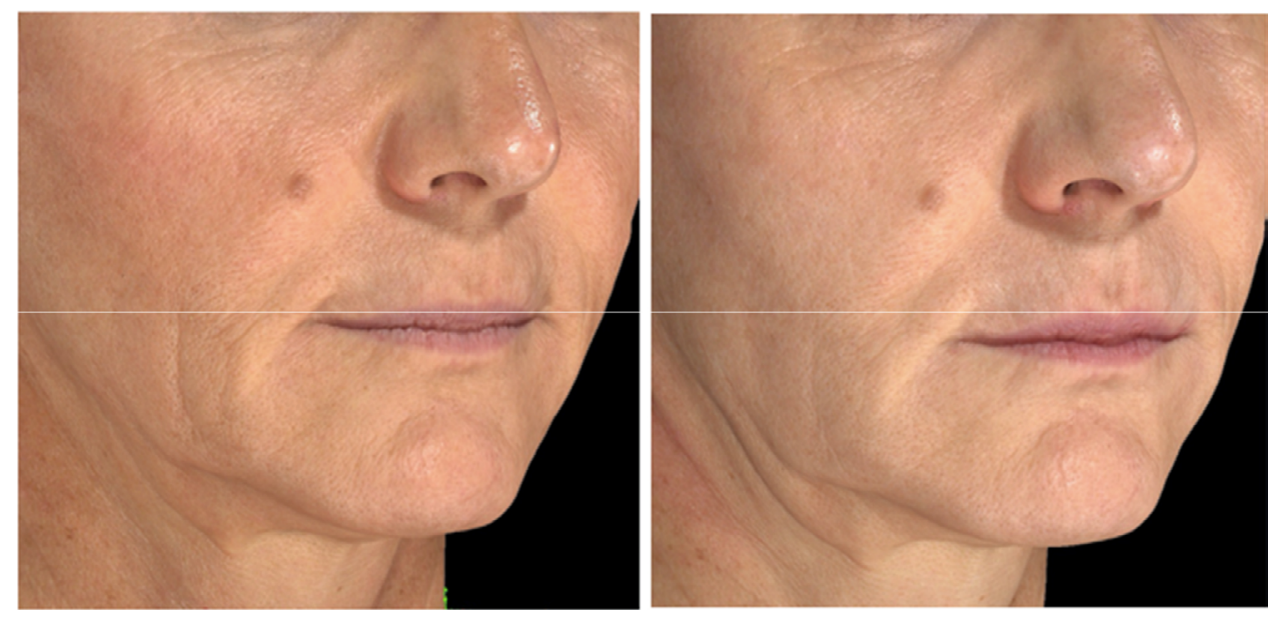

Figure 2 Patient at baseline (left) and 4 weeks after receiving one SP-HA injection in the face (right).

Notes: Patient displays a reduction in her deep wrinkles and fine lines, and the Corneometer assessment revealed an amelioration in her skin's biophysical parameters such as hydration.

Abbreviation: SP-HA, small particle hyaluronic acid.
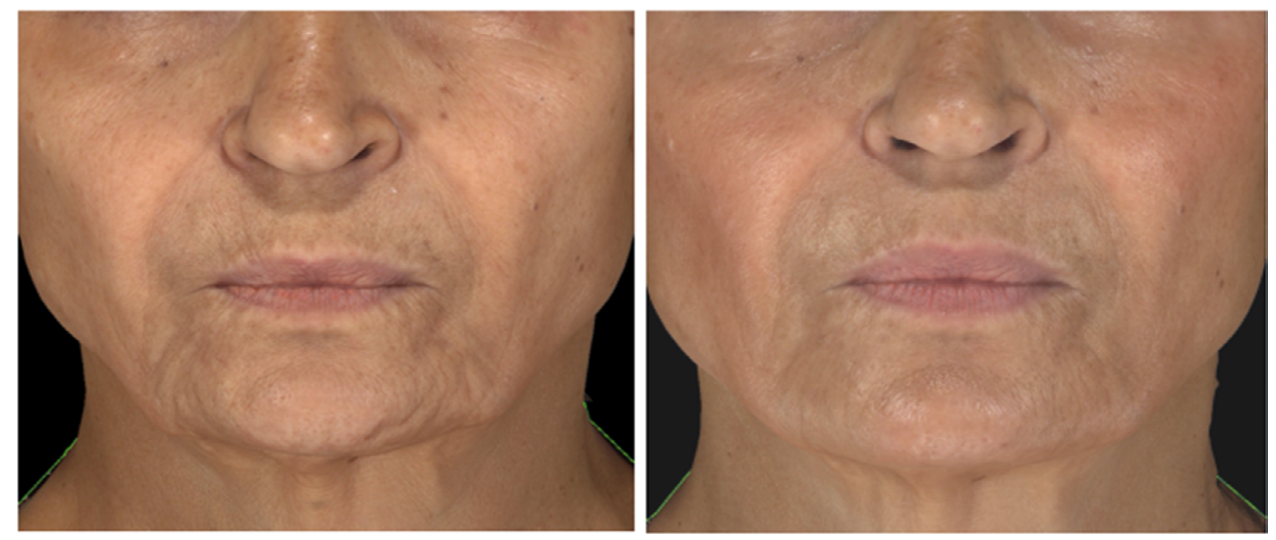

Figure 3 Patient at baseline (left) and Week 8 after receiving two SP-HA injections in the face (right).

Notes: Patient displays a reduction in the lines of her perioral area, and the Courage + Khazaka assessments revealed an amelioration in her skin's biophysical parameters. Abbreviation: SP-HA, small particle hyaluronic acid. 

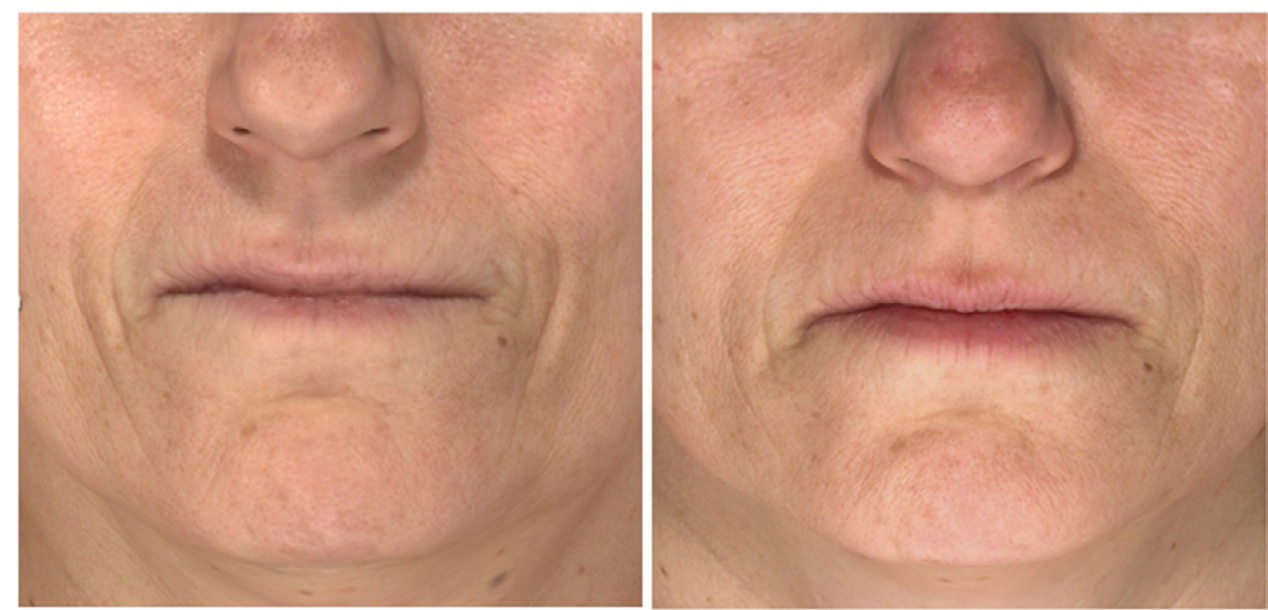

Figure 4 Patient at baseline (left) and Week 12 after receiving three SP-HA injections in the face (right).

Notes: Patient displays a reduction in her moderate to deep lines, and the Courage + Khazaka assessments revealed an amelioration in her skin's biophysical parameters. Abbreviation: SP-HA, small particle hyaluronic acid.

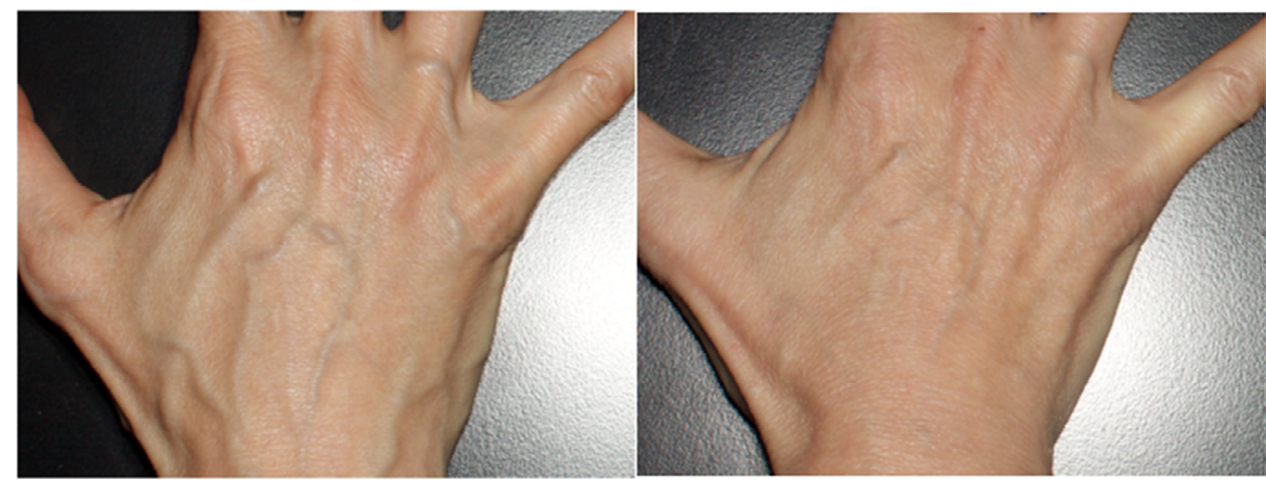

Figure 5 Patient at baseline (left) and Week 12 after receiving three SP-HA injections in the hands (right).

Notes: Patient displays a reduction in the signs of aging in her hands (eg, loss of fat, visible veins, muscles, and tendons), and the Courage + Khazaka assessments revealed an amelioration in her skin's biophysical parameters.

Abbreviation: SP-HA, small particle hyaluronic acid.
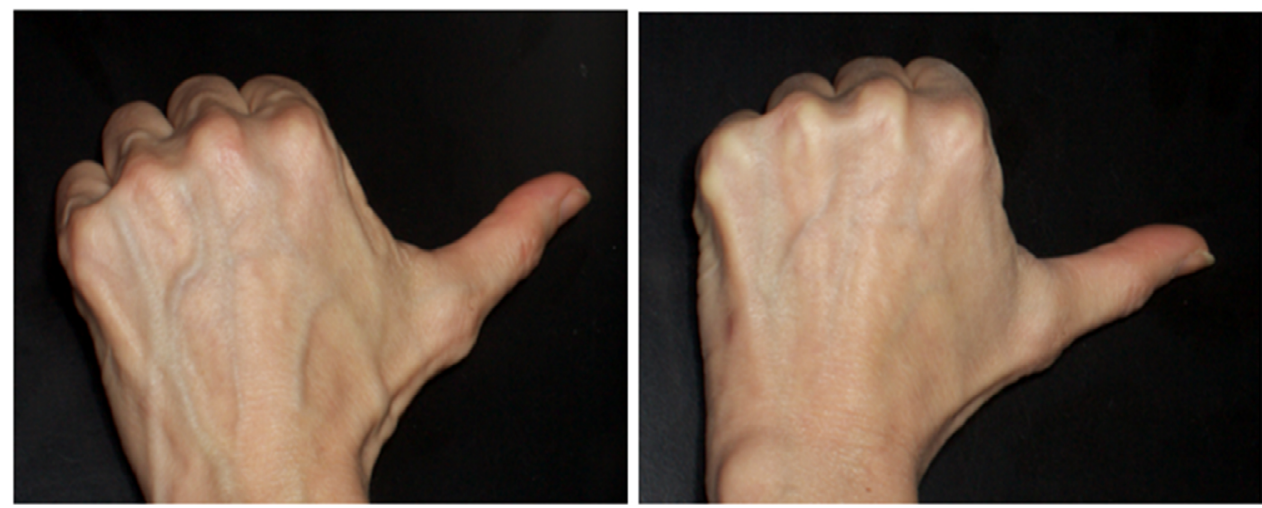

Figure 6 Patient at baseline (left) and Week 12 after receiving three SP-HA injections in the hands (right).

Notes: Patient displays a reduction in the signs of aging in her hands (eg, loss of fat, visible veins, muscles, and tendons), and the Courage + Khazaka assessments revealed an amelioration in her skin's biophysical parameters.

Abbreviation: SP-HA, small particle hyaluronic acid. 
received treatments in multiple areas with the following combinations being present in the sample: $n=8$, face, neck, and hands; $n=6$, face and neck; $n=3$, face and hands; $n=2$, face only; $n=1$, neck and hands. All subjects received bilateral treatments.

\section{Corneometer (hydration)}

Table 1 displays the mean, SDs, and mean differences from baseline for the hydration values, as determined by the Corneometer, for the face, neck, and hands (bilaterally). Face: at Visit 1, mean values were considered to be "dry" (ie, between 30 and 40). At Visits 2, 3, and 4, mean values were considered to be "sufficiently hydrated" (ie, >40), as per manufacturer guidelines. ${ }^{17}$

Neck: For all visits, mean values were considered to be "sufficiently hydrated" (ie, >40), as per manufacturer guidelines. ${ }^{17}$ Hydration values at Visit 3 were significantly higher than during Visit 1 on both sides of the neck $(P=0.045$, right side; $P=0.001$, left side). All other comparisons were not statistically significant $(P>0.05)$.

Hands: At Visit 1, mean values were considered to be "very dry" (ie, <30). At Visits 2, 3, and 4, mean values were considered to be "dry" (ie, between 30 and 40), as per manufacturer guidelines. ${ }^{17}$ Visit 3 was statistically significant to Visit 1 on both the right $(P=0.006)$ and left $(P=0.046)$ sides. Visit 4 was also statistically significant to Visit 1 on both the right $(P=0.013)$ and left sides $(P=0.025)$. All other comparisons were not statistically significant $(P>0.05)$.

\section{Tewameter (trans-epidermal water loss)}

Table 2 displays the mean, SDs, and mean differences from baseline of the TEWL values, as determined by the Tewameter, for the face, neck, and hands (bilaterally).

Face: At all visits, mean values were always below the $25 \mathrm{~g} / \mathrm{h} / \mathrm{m}^{2}$ critical evaporation threshold. There was one case (ie, Visit 2 to Visit 1, right side) where values significantly deviated from baseline $(P=0.044)$, and this was corrected by Visit 3 (Visit 1 to Visit 3, right side $P=0.131$ ). All other comparisons were not statistically significant $(P>0.05)$.

Neck: At all visits, mean values were always below the $25 \mathrm{~g} / \mathrm{h} / \mathrm{m}^{2}$ critical threshold. No significant differences were found between visits $(P>0.05)$.

Hands: At Visit 1 and Visit 2, mean TEWL values were above the $25 \mathrm{~g} / \mathrm{h} / \mathrm{m}^{2}$ critical threshold. At Visit 3 and Visit 4 , mean TEWL values were below the $25 \mathrm{~g} / \mathrm{h} / \mathrm{m}^{2}$ critical threshold. TEWL values at Visit 3 and Visit 4 significantly decreased from Visit $1(P=0.000)$ and Visit $2(P=0.000)$, on both sides. Visit 2 was not statistically significant to Visit 1 , on either side $(P>0.05)$.

\section{$\mathrm{pH}$ meter $(\mathrm{pH})$}

Face: At all visits, mean $\mathrm{pH}$ values fell within normal ranges (ie, 4.0-7.0). There were no statistically significant differences between visits $(P>0.05)$.

Neck: At all visits, mean $\mathrm{pH}$ values fell within normal ranges (ie, 4.0-7.0). Visit 2 was approaching statistical insignificance from Visit 1 on the left side $(P=0.049)$. Visit 4 was statistically different from Visit 1 on both right $(P=0.027)$

Table I Mean, SDs, and mean differences from baseline of the hydration values (as determined by the Corneometer), for the face, neck, and hands (bilateral)

\begin{tabular}{|c|c|c|c|c|c|c|c|c|}
\hline & $\begin{array}{l}\text { VI } \\
\text { Right side }\end{array}$ & $\begin{array}{l}\text { V2 } \\
\text { Right side }\end{array}$ & $\begin{array}{l}\text { V3 } \\
\text { Right side }\end{array}$ & $\begin{array}{l}\text { V4 } \\
\text { Right side }\end{array}$ & $\begin{array}{l}\text { VI } \\
\text { Left side }\end{array}$ & $\begin{array}{l}\text { V2 } \\
\text { Left side }\end{array}$ & $\begin{array}{l}\text { V3 } \\
\text { Left side }\end{array}$ & $\begin{array}{l}\text { V4 } \\
\text { Left side }\end{array}$ \\
\hline \multicolumn{9}{|l|}{ Face } \\
\hline Mean \pm SD & $33.28 \pm 14.82$ & $42.30^{\mathrm{a}} \pm|3.4|$ & $46.77^{\mathrm{b}} \pm 8.31$ & $49.45^{\mathrm{b}} \pm 14.95$ & $39.10 \pm 12.78$ & $46.14^{\mathrm{a}} \pm 13.23$ & $45.11 \pm 8.14$ & $48.31 \pm 15.15$ \\
\hline $\begin{array}{l}\text { Mean dif } \\
\text { from VI }\end{array}$ & - & 9.02 & 13.47 & 16.31 & - & 7.04 & 5.21 & 8.80 \\
\hline \multicolumn{9}{|l|}{ Neck } \\
\hline Mean \pm SD & $48.04 \pm 7.00$ & $52.85 \pm 8.05$ & $57.58^{\mathrm{a}} \pm 10.39$ & $57.02 \pm 5.99$ & $48.44 \pm 7.55$ & $56.45 \pm 9.14$ & $61.87^{\mathrm{b}} \pm 10.87$ & $51.12 \pm 8.82$ \\
\hline $\begin{array}{l}\text { Mean dif } \\
\text { from VI }\end{array}$ & - & 4.36 & 9.48 & 7.76 & - & 7.62 & 13.28 & 0.70 \\
\hline \multicolumn{9}{|l|}{ Hands } \\
\hline Mean & $24.38 \pm 9.90$ & $32.12 \pm 8.02$ & $36.22^{\mathrm{b}} \pm 8.78$ & $35.97^{\mathrm{b}} \pm 8.44$ & $25.84 \pm 8.04$ & $31.98 \pm 7.35$ & $34.68^{\mathrm{b}} \pm 7.30$ & $39.45^{b} \pm 11.10$ \\
\hline $\begin{array}{l}\text { Mean dif } \\
\text { from VI }\end{array}$ & - & 6.14 & 9.54 & 11.02 & - & 4.74 & 6.89 & 11.94 \\
\hline
\end{tabular}

Notes: a'Significance $<0.05$; ${ }^{b}$ significance $<0.005$.

Abbreviation: $\mathrm{V}$, visit. 
Table 2 Mean, SDs, and mean differences from baseline of the trans-epidermal water loss values (as determined by the Tewameter), for the face, neck, and hands (bilateral)

\begin{tabular}{|c|c|c|c|c|c|c|c|c|}
\hline & $\begin{array}{l}\text { VI } \\
\text { Right side }\end{array}$ & $\begin{array}{l}\text { V2 } \\
\text { Right side }\end{array}$ & $\begin{array}{l}\text { V3 } \\
\text { Right side }\end{array}$ & $\begin{array}{l}\text { V4 } \\
\text { Right side }\end{array}$ & $\begin{array}{l}\text { VI } \\
\text { Left side }\end{array}$ & $\begin{array}{l}\text { V2 } \\
\text { Left side }\end{array}$ & $\begin{array}{l}\text { V3 } \\
\text { Left side }\end{array}$ & $\begin{array}{l}\text { V4 } \\
\text { Left side }\end{array}$ \\
\hline \multicolumn{9}{|l|}{ Face } \\
\hline Mean \pm SD & $12.32 \pm 3.09$ & $18.53^{\mathrm{a}} \pm 12.08$ & $13.37 \pm 3.46$ & $12.32 \pm 2.49$ & $14.99 \pm 11.16$ & $17.28 \pm 11.73$ & $12.75 \pm 2.73$ & $12.52 \pm 2.87$ \\
\hline $\begin{array}{l}\text { Mean dif } \\
\text { from VI }\end{array}$ & - & 6.18 & 1.32 & -0.05 & - & 2.26 & -2.96 & -3.47 \\
\hline \multicolumn{9}{|l|}{ Neck } \\
\hline $\begin{array}{l}\text { Mean } \\
\pm S D\end{array}$ & $8.54 \pm 2.11$ & $8.42 \pm 2.68$ & $7.91 \pm 2.13$ & $9.53 \pm 2.16$ & $7.85 \pm 2.38$ & $8.3 \mathrm{I} \pm 2.97$ & $7.24 \pm 2.16$ & $8.63 \pm 1.98$ \\
\hline $\begin{array}{l}\text { Mean dif } \\
\text { from VI }\end{array}$ & - & 0.10 & -0.29 & 1.26 & - & 0.43 & -0.54 & 0.72 \\
\hline \multicolumn{9}{|l|}{ Hands } \\
\hline $\begin{array}{l}\text { Mean } \\
\pm S D\end{array}$ & $24.38 \pm 9.90$ & $30.65 \pm 10.97$ & $12.12^{\mathrm{b}} \pm 5.70$ & $10.11^{b} \pm 3.23$ & $25.84 \pm 8.04$ & $30.34 \pm 10.97$ & $8.92^{\mathrm{b}} \pm 4.12$ & $9.22^{b} \pm 3.2$ \\
\hline $\begin{array}{l}\text { Mean dif } \\
\text { from VI }\end{array}$ & - & 4.67 & -14.55 & -15.84 & - & 3.10 & -18.86 & -17.56 \\
\hline
\end{tabular}

Notes: aSignificance $<0.05$; bsignificance $<0.005$.

Abbreviation: $\mathrm{V}$, visit.

and left $(P=0.019)$ sides, Visit 3 on both right $(P=0.019)$ and left $(P=0.012)$ sides and also from Visit 2 on the right side $(P=0.030)$. All other comparisons were not statistically significant $(P>0.05)$.

Hands: At all visits, mean $\mathrm{pH}$ values fell within normal ranges (ie, 4.0-7.0). There were no statistically significant differences between visits $(P>0.05)$.

Reported AEs were all mild and included the typical and expected incidents associated with an injection procedure, such as temporary erythema, swelling, and bruising associated with the procedure and not to the products themselves. No severe AEs were reported.

\section{Discussion}

Despite the known dehydrating effects of increasing age, ${ }^{11}$ we allowed for the inclusion of wide range of age groups in this study (ie, 30-75 years old). Our significant findings within such a large range of age groups attest to the suitability of SP-HA in young, middle-aged, and older women.

Based on results of the Corneometer, treatment with SP-HA was able to move subjects up to the next hydration level (ie, face went from being dry to moisturized and hands went from being very dry to dry). ${ }^{17}$ For the face, significant results were seen after only one of the three treatment sessions. For the neck and hands, two treatments were needed in order to significantly increase hydration levels. While over the three treatments, there was a general trend in the face, neck, and hands to continuously improve in hydration levels, our findings suggest that two SP-HA treatments may be sufficient to observe clinical effects. As such, a discussion may ensue with the patient with respect to the need for a third treatment. Our finding of a general trend to improve in hydration levels following multiple injections of SP-HA is in alignment with the findings of other researchers, who have investigated the use of HA fillers manufactured using different technologies., ${ }^{5,8,18}$

Throughout the study, the neck had the highest moisture content, followed by the face and hands. It was observed that subjects who were sufficiently hydrated at baseline (ie, $>40$ corneometer value), had hydration scores that remained relatively stable. However, subjects who were dehydrated at baseline (ie, $<20$ corneometer reading) had the greatest change in Corneometer values between visits. This difference in baseline values accounts for the large SD between subjects. Future studies should investigate treatment with SP-HA in a strictly dehydrated population, by using a Corneometer score at baseline of $<20$ as an inclusion criterion, to amplify the observed effects described in the present study. Furthermore, it is expected that in clinical practice, subjects with dehydrated skin are those most likely to experience a significant rejuvenating effect of SP-HA. The ability of SP-HA to increase skin hydration in the face, neck, and hands supports its function as a skin health adjunct in addition to being a HA filler. Our findings contribute to the body of literature increasingly investigating skin rejuvenation using HA, beyond simply treating deep wrinkles and folds. ${ }^{18}$ 
Results from the TEWL analyses revealed that SP-HA was safe and well tolerated and did not damage the stratum corneum's ability to retain moisture or effectively act as a barrier. These data were in accordance with other investigations into the safety of multiple HA injections and their effects on the stratum corneum's barrier function. ${ }^{19}$ This was exemplified by the fact that the treatment regimen did not cause a significant increase in the TEWL scores throughout the study. Additionally, results from TEWL scores on the hands indicate that SP-HA may actually increase the skin's ability to retain moisture. This was evidenced by the fact that after two and three injections of SP-HA, the TEWL scores on the hands significantly decreased to below critical levels. The finding that the stratum corneum had greater water retention following treatment with SP-HA leads to the possibility that HA may reverse possible damage to the skin's water-barrier function vs retaining hydration from the injection of micro-aliquots of product in the deep dermis. Further studies are required to identify the mechanism through which TEWL scores are positively affected by SP-HA.

The slightly variable skin $\mathrm{pH}$ values found in this study fell within the normal acidic range reported in the literature (ie, between $\mathrm{pH} 4.0$ and 7.0), providing evidence that treatment with SP-HA did not disturb the balance of the skin microflora or cause a $\mathrm{pH}$ imbalance. This consistent physiologic marker demonstrates the skin remained within its homeostatic condition throughout treatment. While we found statistically significant differences between the $\mathrm{pH}$ values of some visits, the differences were minimal and would likely not result in clinically significant effects.

A limitation of this study arises from the study design. Given that this was a retrospective chart review, there were inherent disadvantages that could have been avoided with a prospective study. These included possible selection bias, subjects not being adequately matched on certain characteristics, weaknesses in exposure and outcome assessment due to improper recordkeeping, and missing incomplete or poorly documented information. Another limitation was that only one site was used and subjects were usually seen by the same physician at this setting; therefore, possible variations may occur at different sites when physicians with other diagnostic and treatment trends are assessed.

Overall, the findings of this retrospective chart review support that a treatment regimen consisting of three SP-HA injections, spaced 1 month apart, is safe and well tolerated by subjects. SP-HA demonstrated a major hydrating effect (as exemplified by an increase in Corneometer scores) and possible evidence of increasing the skin's ability to retain moisture after only two treatments (as exemplified by a decrease in TEWL scores). Future directions include prospective studies validating these results and further investigating the optimal treatment regimen for improving and/or maintaining skin health with SP-HA.

\section{Statement of institutional review board approval}

In compliance with normative documents governing research with humans, the following study including its protocol, supplemental documents, such as patient consent to photography, and the Principle Investigator and clinical site received unconditional approval from the independent review board "Veritas IRB". A waiver of informed consent was granted by the IRB based on normative guidelines and Health Canada regulations of the Tri-Council Policy Statement articles $3.1-3.5$, as this study posed no risk to the welfare of the participants.

\section{Acknowledgments}

Presented at: IMCAS AMERICAS, March 2017, Cancun, Mexico. This study was funded by an unrestricted educational grant from Galderma Canada.

\section{Author contributions}

AN: principal investigator, article preparation; KME: study coordinator, data abstraction, analyses, article preparation. All authors contributed toward data analysis, drafting and critically revising the paper and agree to be accountable for all aspects of the work.

\section{Disclosure}

The authors wish to disclose the following facts which may be considered as potential conflicts of interest: Dr. Andreas Nikolis is a consultant speaker and research collaborator for Allergan, Galderma, and Merz Pharma. Kaitlyn M Enright reports no conflicts of interest in this work.

\section{References}

1. Brandt FS, Cazzaniga A, Strangman N, Coleman J, Axford-Gatley R. Long-term effectiveness and safety of small gel particle hyaluronic acid for hand rejuvenation. Dermatol Surg. 2012;38(7 Pt 2):1128-1135.

2. Bertucci V, Lynde CB. Current Concepts in the Use of Small-Particle Hyaluronic Acid. Plast Reconstr Surg. 2015;136(5 Suppl):S132-S138.

3. American Society for Dermatologic Surgery. ASDS Survey on Dermatologic Procedures. Available from: https://www.asds.net/_Media. aspx?id=7744. Accessed May 8, 2017.

4. Brandt F, Bassichis B, Bassichis M, O'Connell C, Lin X. Safety and effectiveness of small and large gel-particle hyaluronic acid in the correction of perioral wrinkles. J Drugs Dermatol. 2011;10(9):982-987.

5. Kerscher M, Bayrhammer J, Reuther T. Rejuvenating influence of a stabilized hyaluronic acid-based gel of nonanimal origin on facial skin aging. Dermatol Surg. 2008;34(5):720-726. 
6. Williams S, Tamburic S, Stensvik H, Weber M. Changes in skin physiology and clinical appearance after microdroplet placement of hyaluronic acid in aging hands. $J$ Cosmet Dermatol. 2009;8(3):216-225.

7. Kim J. Effects of injection depth and volume of stabilized hyaluronic acid in human dermis on skin texture, hydration, and thickness. Arch Aesth Plast Surg. 2014;20(2):97-103.

8. Streker M, Reuther T, Krueger N, Kerscher M. Stabilized hyaluronic acid-based gel of non-animal origin for skin rejuvenation: face, hand, and décolletage. J Drugs Dermatol. 2013;12(9):990-994.

9. Gubanova EI, Starovatova PA, Rodina MY, Gubanova EI, Starovatova PA. 12-month effects of stabilized hyaluronic acid gel compared with saline for rejuvenation of aging hands. J Drugs Dermatol. 2015;14(3):288-295.

10. Hadi H, Awadh AI, Hanif NM, Md Sidik NF, Mohd Rani MR, Suhaimi MS. The investigation of the skin biophysical measurements focusing on daily activities, skin care habits, and gender differences. Skin Res Technol. 2016;22(2):247-254.

11. Darlenski R, Sassning S, Tsankov N, Fluhr JW. Non-invasive in vivo methods for investigation of the skin barrier physical properties. Eur J Pharm Biopharm. 2009;72(2):295-303.

12. Berardesca E, Maibach H. Ethnic skin: overview of structure and function. J Am Acad Dermatol. 2003;48(6 Suppl):S139-S142.
13. Wolf R, Tur E, Wolf D, Landau M. The effect of smoking on skin moisture and on surface lipids. Int J Cosmet Sci. 1992;14(2):83-88.

14. du Plessis J, Stefaniak A, Eloff F, et al. International guidelines for the in vivo assessment of skin properties in non-clinical settings: Part 2. transepidermal water loss and skin hydration. Skin Res Technol. 2013;19(3):265-278.

15. Firooz A, Gorouhi F, Davari P, et al. Comparison of hydration, sebum and $\mathrm{pH}$ values in clinically normal skin of patients with atopic dermatitis and healthy controls. Clin Exp Dermatol. 2007;32(3):321-322.

16. Kim SA, Kim BR, Chun MY, Youn SW. Relation between $\mathrm{pH}$ in the Trunk and Face: Truncal $\mathrm{pH}$ Can Be Easily Predicted from Facial pH. Ann Dermatol. 2016;28(2):216.

17. Courage W, Khazaka G. Instruction Manual. Available from: http:// www.courage-khazaka.de/index.php/en/all-downloads/downloads-en. Accessed May 8, 2017.

18. Seok J, Hong JY, Choi SY, Park KY, Kim BJ. A potential relationship between skin hydration and stamp-type microneedle intradermal hyaluronic acid injection in middle-aged male face. J Cosmet Dermatol. 2016;15(4):578-582.

19. Laurino C, Palmieri B, Coacci A, Efficacy CA. Efficacy, Safety, and Tolerance of a New Injection Technique for High- and Low-MolecularWeight Hyaluronic Acid Hybrid Complexes. Eplasty. 2015;15:46.
Clinical, Cosmetic and Investigational Dermatology

\section{Publish your work in this journal}

Clinical, Cosmetic and Investigational Dermatology is an international, peer-reviewed, open access, online journal that focuses on the latest clinical and experimental research in all aspects of skin disease and cosmetic interventions. This journal is included on PubMed. The manuscript management system is completely online

\section{Dovepress}

and includes a very quick and fair peer-review system, which is all easy to use. Visit http://www.dovepress.com/testimonials.php to read real quotes from published authors 